\title{
DEVELOPMENT OF THE BRIEF BEDSIDE DYSPHAGIA SCREENING TEST - REVISED: A CROSS-SECTIONAL CZECH STUDY
}

\author{
Petra Mandysová ${ }^{1,2}$, Edvard Ehler ${ }^{2,1}$, Jana Škvrňáková ${ }^{1,3}$, Michal Černy̆́, Iva Bártováa ${ }^{3}$, Arnošt Pellant ${ }^{1,3}$
}

Faculty of Health Studies, University of Pardubice, Czech Republic ${ }^{1}$; Department of Neurology, Pardubice Hospital, Hospitals of the Pardubice Region, Czech Republic ${ }^{2}$; Department of Otorhinolaryngology and Head and Neck Surgery, Pardubice Hospital, Hospitals of the Pardubice Region, Czech Republic ${ }^{3}$; Department of Otorhinolaryngology and Head and Neck Surgery, University Hospital Hradec Králové, Charles University in Prague, Faculty of Medicine in Hradec Králové, Czech Republic ${ }^{4}$

Summary: Aim: The purpose of this study was to develop a revised version of the Brief Bedside Dysphagia Screening Test for determining penetration/aspiration risk in patients prone to dysphagia. The priority was to achieve high sensitivity and negative predictive value. Methods: The study screeners conducted bedside assessment of the swallowing function in 157 patients with a neurological (mainly stroke) or an ear, nose, and throat diagnosis (mainly head and neck cancer). The results were compared with a gold standard, flexible endoscopic examination of swallowing. Results: For the neurological subgroup ( $\mathrm{N}=106)$, eight statistically significant bedside assessment items were combined into the Brief Bedside Dysphagia Screening Test-Revised (BBDST-R). Cut-off score 1 produced the highest sensitivity $(95.5 \%$; $95 \%$ confidence interval CI [CI]: 84.9-98.7\%) and negative predictive value (88.9\%; 95\% CI 67.2-96.9\%). Conclusion: The BBDST-R is suitable for dysphagia screening in departments caring for patients with neurological conditions.

Keywords: Dysphagia; Screening; Swallowing Disorders; Symptom assessment

\section{Introduction}

Dysphagia (impaired swallowing) is a potentially serious health care problem that can lead to various complications. They can include malnutrition and dehydration due to decreased efficacy of swallowing (1) and aspiration, pneumonia (2), and death (3) due to impaired safety of swallow (1).

Dysphagia is a relatively frequent condition. However, the reported prevalence rates vary, mainly due to the exact definition of dysphagia (4) and the used diagnostic instrument (5). It is estimated to occur in up to $52.7 \%$ of the elderly (2), 44-53.6\% of patients with stroke $(6,7)$, up to $32 \%$ of patients with Parkinson's disease (8), and $48.4 \%$ of patients with head and neck cancer (9).

A timely and accurate diagnosis of dysphagia is an important prerequisite for planning and implementing effective compensatory and rehabilitative interventions (3). In many settings, bedside dysphagia screening is an important first step in the diagnostic process (3). Ideally, dysphagia screening is a quick, minimally invasive procedure that enables the identification of patients who need further assessment $(3,10)$.

Numerous dysphagia screening instruments (DSI) exist, and several literature reviews focusing on their quality have been published (3). Most of them have focused on dyspha- gia screening in patients with neurological disorders $(3,11)$, mainly stroke $(12,13)$. Some DSI are intended for patients with head and neck cancer (14) or for heterogeneous patient groups $(15,16)$.

Despite the plethora of the published DSI, none of them has been endorsed as the most useful screening method (11), partly due to a lack of consensus as to what constitutes a good DSI (10). It should be valid; therefore, many studies aiming to develop DSI use an "objective" reference test (a gold standard) that determines the presence or absence of dysphagia (10). The most widely recognized gold standards are videofluoroscopy and flexible endoscopic evaluation of swallowing (FEES) (3). The use of a gold standard enables comparisons with bedside assessments and subsequent selection of assessment items for the DSI (17).

A good DSI should be sensitive to the condition being observed (dysphagia). In fact, obtaining the highest possible sensitivity is a priority of most DSI (10). The reason is that missing (not capturing) someone who has dysphagia can lead to serious complications (10). Conversely, high specificity, i.e. correctly ruling out the patients who do not have dysphagia, is secondary as the consequences of incorrectly identifying someone as having a swallowing problem are not as serious (10). Other experts believe that in addition to high sensitivity, a good DSI should have high negative predictive value (12). It is important that patients with a normal 
screening result have normal swallowing function (which is reflected in high negative predictive value) because patients with a normal screening result are not referred for a detailed clinical swallow examination.

The Brief Bedside Dysphagia Screening (BBDS) Test was the result of a study aiming to a) develop a DSI while using FEES (the gold standard) on all enrolled patients, and b) enroll patients with various conditions predisposing to penetration/aspiration (17). The BBDS Test had high sensitivity $(95.2 \%$; 95\% confidence interval $[\mathrm{CI}]: 77.3-99.2 \%)$ and negative predictive value $(93.3 \%$; 95\% CI: 70.2-98.8\%) in the neurological subgroup (17). However, both parameters were lower in the ear, nose, and throat (ENT) subgroup (17). Consequently, overall sensitivity $(87.1 \%$; 95\% CI: $71.1-94.9 \%)$ and negative predictive value $(81.0 \%$; $95 \%$ CI: $60.0-92.3 \%$ ) were lower (17). Clearly, the heterogeneity of the patients was an impediment to achieving high diagnostic parameters.

Despite abundant literature regarding the requirements for DSI, the issue of an a priori sample size calculation in studies that aim to develop a DSI is rarely discussed (18). Yet, it is important to calculate the number of subjects needed to define an expected level of the sought-after diagnostic parameter, for example sensitivity, together with the precision of that estimate (that is, the confidence intervals) (19). In other words, researchers should aim for a high point estimate of the diagnostic parameter as well as a reasonably narrow CI, which reflects greater precision of the estimate than wider CI (18).

The aim of the study was to produce a revised version of the mentioned BBDS Test by expanding Mandysova et al.'s (17) pilot study. The revised study was based on the same premise and had the same aim as the pilot study; the patient inclusion criteria and data collection method did not change. However, we present a novel approach in four areas. First, criteria were developed for assessing whether the patient group was "sufficiently homogenous" - this became a requirement for the development of a generic DSI. If this were not the case, the aim would be to develop a DSI for only the bigger patient subgroup (neurological or ENT). Second, the sought-after diagnostic parameters were clearly spelled out: priority was given to achieving the highest possible sensitivity and negative predictive value. Third, an expected level of sensitivity and the precision of that estimate were set a priori and the number of subjects needed was calculated. Fourth, part of the aim was to identify the optimal cut-off point of the instrument by calculating diagnostic parameters for all the possible scores.

\section{Materials and Methods}

Design. This cross-sectional study was conducted in a regional Czech hospital in the department of neurology, ENT, and geriatrics, with a purposive sample of 157 acutely or chronically ill patients (inpatients and outpatients) who were prone to dysphagia based on their primary neurological or ENT diagnosis. It was part of a larger study that started in 2009 and spanned 60 months; this particular phase lasted 31 months.

Two sets of investigations were carried out: a detailed bedside assessment of the swallowing function by a nurse screener and FEES by a physician. Based on a comparison of these two investigations, selected items of the bedside assessment were combined into the BBDS Test-Revised (BBDST-R). A preliminary analysis of the results, leading to the development of the BBDS Test, was conducted after the first 18 months of data collection and was described by Mandysova et al. (17). This period was the pilot study; the collected data were incorporated into the revised study.

Subjects. Inpatients were recruited via nurses or physicians on the wards; outpatients were recruited during their visits to the dysphagia clinic (17). The inclusion criteria were: prone to dysphagia based on the primary neurological or ENT diagnosis; receiving care in one of the mentioned departments; medically stable (not receiving care in the intensive care unit); sufficiently alert (able to follow simple commands); able to maintain a sitting position; able to sign an informed consent (17).

Definitions. Sufficient homogeneity was defined in the following way: the difference between relative frequencies of abnormal results in the neurological subgroup and those in the ENT subgroup should be $<5 \%$ for $>50 \%$ of the bedside assessment items and FEES (criterion 1) and simultaneously, it should be $<10 \%$ for all bedside assessment items and FEES (criterion 2). If both criteria were not met, a DSI would be developed for only the bigger patient subgroup (neurological or ENT).

Sample size calculation. Flahault et al.'s (20) guidelines for sample size calculation in diagnostic test studies were followed to ensure a given precision of the sensitivity estimate. The first step entails an assumption on the expected value of the new diagnostic test sensitivity (20). Next, the minimum acceptable lower confidence limit is determined, together with the required probability that this limit is not violated (20). The minimal sample size for the group of "cases" $\left(N_{\text {cases }}\right)$ is read from the provided tables, and the corresponding number of "controls" $\left(N_{\text {controls }}\right)$ is obtained from an equation that assumes disease prevalence $($ Prev $)<50 \%$ :

$N_{\text {controls }}=N_{\text {cases }}[(1-$ Prev $) /$ Prev $]$ (Equation 1) (20). For the purpose of this study, the disease (dysphagia) prevalence assumption was based on the relative frequency of abnormal FEES in the pilot study, which was $35.6 \%$ for all patients and $29.2 \%$ for the neurological subgroup (17). Therefore, the above Equation 1 could be used. For Prev $>50 \%$, Flahault et al. (20) recommend using the same equation with $N_{\text {controls }}$ in place of $N_{\text {cases }}$ (Equation 2), which would be relevant for the development of an ENT instrument based on dysphagia prevalence in the ENT subgroup (66.7\%) in the pilot study (17). Expected sensitivity of the instrument was set at $95 \%$, and the lower $95 \%$ confidence limit was set at $75 \%$ with 0.95 probability. $N_{\text {cases }}(=34)$ was obtained from the table and $N_{\text {controls }}(=60)$ from Equation $1(20)$; the total required 
sample size was 94 patients. A 20\% reserve was planned for patients who might drop out as recommended by Bochmann et al. (18) or whose data would be eliminated in the data analysis phase. Therefore, the necessary sample size was 112-113 patients. Since the type of the DSI (generic versus for a homogenous patient group) was determined only in the data analysis phase, patient enrollment was to continue until one of the two subgroups (rather than the entire group) included 112-113 patients.

Instruments. For the data collection phase, a detailed 32-item bedside assessment instrument was developed. The instrument entailed physical assessment (20 items) and a swallow test (12 items). Twenty-four of the items were based on the Massey Bedside Swallowing Screen (21) and the Gugging Swallowing Screen (GUSS) (22). Additional 8 physical assessment items were based on discussions with dysphagia experts, as particularized by Mandysova et al. (17). Physical assessment focused mainly on the reflexes and the motor function of the muscles involved in swallowing (17). The swallow test comprised three sequential steps: swallowing 1) a thick liquid (pudding-like consistency, four teaspoons), 2) a thin liquid (four teaspoons), and 3 ) a thin liquid (60 mL, drinking from a cup) (17). Administering a thick liquid before a thin liquid was in accordance with the GUSS (22), and thin liquid testing was congruent with Massey and Jedlicka's procedure $(17,21)$. Testing was terminated if the patient experienced cough, choking, wet/ gurgly voice, or the liquid dripping from the mouth during and for up to one minute following each step of the swallow test. The technique of the physical assessment and swallow test was described in depth by Mandysova et al. (17).

Penetration Aspiration Scale (PAS) was used to score selected aspects of swallowing assessed by the gold standard, FEES (23). PAS is an eight-point ordinal scale quantifying penetration (passage of material into the larynx to the level of the vocal folds) and aspiration (passage of material below the level of the vocal folds) (23). Furthermore, it reflects whether or not the material is expelled (23).

Procedure. Three investigators performed the bedside assessment. Two were university-educated nurses with extensive advanced practice experience in the field. The third one was a master-level nursing student and was involved for only a 12-month period between $03 / 2010$ and 02/2011, which corresponded to the period of her master's degree project. Two specially trained physicians performed FEES.

The aim was to perform both assessments as soon as the patient met the inclusion criteria. At the same time, it was ensured that the period between both assessments was as short as possible. For the neurological subgroup $(\mathrm{N}=106)$, the period was, on average, 1.22 days; for $96(90.6 \%)$ patients, the period was $\leq 24$ hours. For the ENT subgroup $(\mathrm{N}=38)$, the period was, on average, 2.84 days; for $25(65.7 \%)$ patients, it was $\leq 24$ hours. The sequence of the two assessments depended mainly on the availability of the personnel involved in the study.
Some patients did not complete certain bedside assessment items (patient refused or did not understand more detailed instructions; the swallow test was terminated). Such occurrences were treated as missing items. Conversely, patients who did not complete both the bedside assessment and FEES were excluded.

Data analysis. Each bedside assessment item result was dichotomized: normal/negative versus abnormal/positive, and so were the PAS scores: $1=$ normal $/$ negative versus 2-8 $=$ abnormal/positive. Patients with a normal FEES and bedside assessment in all 32 items were excluded from further analysis because their data would not contribute to the explanation of observed variation in the results.

To address the issue of sufficient homogeneity, absolute and relative frequencies of abnormal bedside assessment and FEES results in both patient subgroups (neurological and ENT) were calculated and compared, using the two mentioned criteria. This step determined whether all the subsequent steps of data analysis should use the results of all the patients (for the development of a generic DSI) or the results of only the bigger patient subgroup.

Next, the results between individual bedside assessment items and FEES were compared, using the association coefficient $\varphi$ (phi). The $\varphi$ coefficient indicates the tightness and direction (positive or negative) of the association between two dichotomous variables (24). Zero indicates no association and \pm 1 indicates a perfect association if the frequency of both variables in a $2 \times 2$ contingency table is evenly split (24). Calculations were performed with SPSS 19.0 statistical software (IBM SPSS, Inc., Chicago, Illinois). Bedside assessment items with a positive and statistically significant association ( $p$-value $\leq 0.05)$ with FEES were combined into a DSI if the bedside assessment item contained $\leq 5 \%$ of missing values, as recommended by Schafer (25).

The total score of the developed DSI was obtained by summing up the scores of all of its items (normal/negative item $=0 ;$ abnormal $/$ positive item $=1)$. The total score was dichotomized (normal/"pass" versus abnormal/"fail") using all the possible cut-off scores. Next, it was examined which cut-off score resulted in the highest sensitivity and negative predictive value. A $2 \times 2$ contingency table was created, and using the patients' results, the number of truly positive (FEES and bedside assessment abnormal), truly negative (FEES and bedside assessment normal), falsely positive (FEES normal and bedside assessment abnormal), and falsely negative (FEES abnormal and bedside assessment normal) cases was determined for each possible cut-off score. Subsequently, sensitivity, specificity, and positive and negative predictive values were computed using the Clinical Calculator 1 (26). For sensitivity, the results were compared with the a priori determined expected sensitivity and its lower $95 \%$ confidence limit.

Ethical considerations. The study was conducted according to the Declaration of Helsinki and was approved by the hospital ethics committee. Prior to enrolling the 
participants, the researchers were required to obtain written informed consent while preventing undue influence on potential participants.

\section{Results}

Of the 180 patients who were approached, 2 refused to participate, 15 did not undergo FEES ( 7 refused; 8 were not transported for the examination by the staff for various reasons), and 6 did not undergo detailed bedside assessment ( 1 refused; 1 died; 4 were discharged). The mean age of the remaining 157 patients ( 99 men; 58 women) was $67.6 \pm 13.6$ years (range 21-91); 107 were inpatients and 50 were outpatients. One hundred and twelve patients had a neurological condition (stroke 54; myasthenia gravis 30; amyotrophic lateral sclerosis 6; cranial nerve palsy 5; Parkinson's disease 4 and other conditions 13). Forty-five patients had an ENT condition (head or neck cancer 23; dysphagia, unspecified 7; inflammation or infection 4 and other conditions 11). Of these, 12 (6 with a neurological and 6 with an ENT condition) were excluded from subsequent data analysis because all their results were normal. Another patient from the ENT subgroup was excluded as he obtained no PAS score due to a permanent separation of the airway from the oral and pharyngeal pathway after a laryngectomy.

Of the remaining 144 patients, 67 (46.5\%) had an abnormal FEES result: $44(41.5 \%)$ of patients from the

Tab. 1: Bedside assessment item results with a statistically significant association to FEES.

\begin{tabular}{|l|c|c|c|}
\hline Bedside assessment item* & $\begin{array}{c}\text { Number } \\
\text { of assessed } \\
\text { patients } \\
\text { n (\%) }\end{array}$ & $\boldsymbol{\varphi}$ & P-value \\
\hline Absence of voluntary cough & $103(97.2)$ & 0.354 & $<0.001$ \\
\hline Dysarthria & $100(94.3)$ & 0.436 & $<0.001$ \\
\hline $\begin{array}{l}\text { Shoulder shrug asymmetrical } \\
\text { and/or of abnormal strength }\end{array}$ & $102(96.2)$ & 0.279 & 0.005 \\
\hline $\begin{array}{l}\text { Thin liquid (drinking): } \\
\text { dripping from the mouth** }\end{array}$ & $71(67.0)$ & 0.303 & 0.011 \\
\hline $\begin{array}{l}\text { Tongue asymmetrical } \\
\text { and/or of abnormal strength }\end{array}$ & $101(95.3)$ & 0.239 & 0.016 \\
\hline Thick liquid: choking & $101(95.3)$ & 0.236 & 0.018 \\
\hline Aphasia & $105(99.1)$ & 0.227 & 0.020 \\
\hline $\begin{array}{l}\text { Facial muscles asymmetrical } \\
\text { and/or of abnormal strength }\end{array}$ & $104(98.1)$ & 0.220 & 0.025 \\
\hline $\begin{array}{l}\text { Thin liquid (drinking): } \\
\text { cough** }\end{array}$ & $74(69.8)$ & 0.255 & 0.029 \\
\hline Unable to clench the teeth & $104(98.1)$ & 0.197 & 0.045 \\
\hline
\end{tabular}

* Assessed items are described in terms of their abnormal result. ** The item was not included in the dysphagia screening instrument due to significantly $>5 \%$ of missing values (percentage of missing values $=100-$ number of assessed patients, in \%). $\boldsymbol{\varphi}$ : association coefficient phi, FEES: flexible endoscopic examination of swallowing. neurological and $23(60.5 \%)$ from the ENT subgroup. The relative frequency of abnormal results between both subgroups differed by the predetermined $<5 \%$ on $16(50 \%)$ of the individual bedside assessment items and by the predetermined $<10 \%$ on 25 bedside assessment items. The most pronounced difference was noted on items "symmetry/ strength of the facial muscles" (abnormal: 55.8\% neurological versus $28.9 \%$ ENT subgroup; difference $26.9 \%$ ), "voice change" (79.2\% vs. 52.6\%; difference $26.6 \%)$, "dysarthria" (56.0\% vs. $36.8 \%$; difference $19.2 \%$ ), and "aphasia" (19.0\% vs. $2.6 \%$; difference $16.4 \%$ ). On FEES, the difference between the two subgroups was 19\%. Because the homogeneity criteria were not met, all subsequent calculations were based on the results of the bigger, neurological subgroup $(\mathrm{N}=106)$.

A comparison of the results using the $\varphi$ coefficient showed a statistically significant, positive association ( $p$-value $\leq 0.05$ ) between FEES and 10 bedside assessment items (Table 1). Two of the bedside assessment items contained significantly $>5 \%$ of missing values. Therefore, only the remaining 8 were combined into an 8 -item DSI (Figure 1).

The diagnostic parameters of the BBDST-R depended on the number of true and false negative and true and false positive results. This in turn depended on the relevant cut-off score (Table 2). Cut-off score 1 produced $95.5 \%$ sensitivity (95\% CI 84.9-98.7\%) and 88.9\% negative predictive value $(95 \%$ CI $67.2-96.9 \%)$. The higher the cut-off score, the lower the value of both of these parameters.

\begin{tabular}{|l|l|}
\hline Bedside assessment item & Score $^{* *}$ \\
\hline 1. Presence of voluntary cough & \\
\hline 2. Ability to clench the teeth & \\
\hline 3. The tongue is symmetrical and strong & \\
\hline 4. The facial muscles are symmetrical and strong & \\
\hline 5. Shoulder shrug is symmetrical and strong & \\
\hline 6. Presence of dysarthria & \\
\hline 7. Presence of aphasia & \\
\hline 8. Thick liquid: cough * & \\
\hline Total: $* * *$ & \\
\hline
\end{tabular}

Fig. 1: The Brief Bedside Dysphagia Screening Test-Revised.

* Four teaspoons (pudding-like consistency) are given and the patient is observed for $\leq 1$ minute afterwards. ** Each abnormal item $=1$ point; each normal item $=0$ points. Abnormal finding: "No" for items 1-5 and "Yes" for items 6-8. *** Cut-off score $=1$ (test negative if total score $=0$, positive if total score $\geq 1$ ).

\section{Discussion}

A comparison of relative frequencies of abnormal results obtained for the two subgroups revealed that neither of the two predetermined criteria was met. The two subgroups differed by $16.4-26.9 \%$ on 4 bedside assessment items. In all four cases, the frequency of abnormal results was higher in the neurological subgroup. Moreover, they differed by $19 \%$ 
Tab. 2: Diagnostic parameters of the Brief Bedside Dysphagia Screening Test-Revised.

\begin{tabular}{|c|c|c|c|c|c|c|c|}
\hline \multicolumn{8}{|c|}{ BBDST-R $(n=106)$} \\
\hline $\mathbf{T N}$ & FN & TP & FP & $\begin{array}{l}\text { Sensitivity } \\
\text { (95\% CI) }\end{array}$ & $\begin{array}{l}\text { Specificity } \\
\text { (95\% CI) }\end{array}$ & $\begin{array}{c}\text { NPV } \\
(95 \% \text { CI })\end{array}$ & $\begin{array}{c}\text { PPV } \\
(95 \% \text { CI })\end{array}$ \\
\hline \multicolumn{4}{|c|}{$\mathbf{N}$} & \multicolumn{4}{|c|}{$\%$} \\
\hline \multicolumn{8}{|c|}{ Cut-off score 1} \\
\hline 16 & 2 & 42 & 46 & $\begin{array}{c}95.5 \\
(84.9-98.7)\end{array}$ & $\begin{array}{c}25.8 \\
(16.6-37.9)\end{array}$ & $\begin{array}{c}88.9 \\
(67.2-96.9)\end{array}$ & $\begin{array}{c}47.7 \\
(37.6-58.0)\end{array}$ \\
\hline \multicolumn{8}{|c|}{ Cut-off score 2} \\
\hline 32 & 7 & 37 & 30 & $\begin{array}{c}84.1 \\
(70.6-92.1)\end{array}$ & $\begin{array}{c}51.6 \\
(39.4-63.6)\end{array}$ & $\begin{array}{c}82.1 \\
(67.3-91.0)\end{array}$ & $\begin{array}{c}55.2 \\
(43.4-66.5)\end{array}$ \\
\hline \multicolumn{8}{|c|}{ Cut-off score 3} \\
\hline 44 & 12 & 32 & 18 & $\begin{array}{c}72.7 \\
(58.2-83.7)\end{array}$ & $\begin{array}{c}71.0 \\
(58.7-80.8)\end{array}$ & $\begin{array}{c}78.6 \\
(66.2-87.3)\end{array}$ & $\begin{array}{c}64.0 \\
(50.1-75.9)\end{array}$ \\
\hline \multicolumn{8}{|c|}{ Cut-off score 4} \\
\hline 55 & 25 & 19 & 7 & $\begin{array}{c}43.2 \\
(29.7-57.8)\end{array}$ & $\begin{array}{c}88.7 \\
(78.5-94.4)\end{array}$ & $\begin{array}{c}68.8 \\
(57.9-77.8)\end{array}$ & $\begin{array}{c}73.1 \\
(53.9-86.3)\end{array}$ \\
\hline \multicolumn{8}{|c|}{ Cut-off score 5} \\
\hline 61 & 32 & 12 & 1 & $\begin{array}{c}27.3 \\
(16.3-41.8)\end{array}$ & $\begin{array}{c}98.4 \\
(91.4-99.7)\end{array}$ & $\begin{array}{c}65.6 \\
(55.5-74.5)\end{array}$ & $\begin{array}{c}92.3 \\
(66.7-98.6)\end{array}$ \\
\hline \multicolumn{8}{|c|}{ Cut-off score 6} \\
\hline 61 & 40 & 4 & 1 & $\begin{array}{c}9.1 \\
(2.4-14.6)\end{array}$ & $\begin{array}{c}98.4 \\
(93.1-99.8)\end{array}$ & $\begin{array}{c}60.4 \\
(47.1-63.4)\end{array}$ & $\begin{array}{c}80.0 \\
(37.6-96.4)\end{array}$ \\
\hline \multicolumn{8}{|c|}{ Cut-off score 7} \\
\hline 62 & 43 & 1 & 0 & $\begin{array}{c}2.3 \\
(0.1-13.5)\end{array}$ & $\begin{array}{c}100.0 \\
(92.7-100.0)\end{array}$ & $\begin{array}{c}59.0 \\
(49.0-68.4)\end{array}$ & $\begin{array}{c}100.0 \\
(5.5-100.0)\end{array}$ \\
\hline \multicolumn{8}{|c|}{ Cut-off score 8} \\
\hline 62 & 44 & 0 & 0 & $\begin{array}{c}0.0 \\
(0.0-10.0)\end{array}$ & $\begin{array}{c}100.0 \\
(92.7-100.0)\end{array}$ & $\begin{array}{c}58.5 \\
(48.5-67.9)\end{array}$ & -* \\
\hline
\end{tabular}

* The calculation could not be performed because the value entered included an instance of zero.

BBDST-R: Brief Bedside Dysphagia Screening Test-Revised, CI: confidence interval, FN: false-negative, FP: false-positive, NPV: negative predictive value, PPV: positive predictive value, TN: true-negative, TP: true-positive.

on FEES. In contrast, however, the frequency of abnormal FEES findings was higher in the ENT subgroup. Evidently, the sufficient homogeneity criteria were not met, and it was not realistic to develop a generic DSI. Because this conclusion was not known a priori, patient enrollment continued until the planned sample size was achieved for the bigger, neurological subgroup $(\mathrm{N}=112)$.

In the neurological subgroup, two bedside assessment items with a statistically significant relationship to FEES contained $>5 \%$ of missing values: "thin liquid (drinking): dripping from the mouth" (67\% patients assessed) and "thin liquid (drinking): cough" (69.8\%) (Table 1). This was mainly because drinking thin liquid was the third step of the swallow test, which was not performed if the test was abnormal in the two preceding steps. Consequently, only one consistency of the liquid (thick) was included in the DSI.
The thick liquid item is consistent with the first step of the Direct Swallowing Test contained in the GUSS (22). Like the original BBDS Test, the BBDST-R contains 8 items; however, two BBDS items ("thick liquid: choking" and "thick liquid: dripping from the mouth") (17) were replaced by "voluntary cough" and "aphasia".

Since our priority was to achieve high sensitivity and negative predictive value, the BBDST-R has the best performance for cut-off score 1: sensitivity $95.5 \%$ (95\% CI $84.9-98.7 \%)$ and negative predictive value $88.9 \%(95 \%$ CI 67.2-96.9\%) (Table 2). Thus, the BBDST-R is negative (normal) for total score $=0$ and positive (abnormal) for total score $=1-8$ (Figure 1). The resultant sensitivity $(95.5 \%)$ is better than the a priori determined expected sensitivity $(95 \%)$ and so is its lower $95 \%$ confidence limit (84.9\% versus the a priori determined $75 \%$ ), which was probably due 
to a larger patient sample ( $\mathrm{N}=106$ after data reduction) than required $(\mathrm{N}=94)$ based on Flahault et al.'s Equation $1(20)$. Flahault et al.'s disease prevalence assumption (Prev $<50 \%$ ) (20) was satisfied: for the neurological subgroup, FEES was abnormal in $41.5 \%$ of the cases.

A systematic review of 14 currently available screenings for neurological patients concluded that only four DSI were of sufficient methodological quality (3). One of them is Martino et al.'s (27) Toronto Bedside Swallowing Screening Test (TOR-BSST). Compared with our BBDST-R, the TORBSST has similar sensitivity $(91.3 \%$; 95\% CI 71.9-98.7\%) and negative predictive value in acute $(93.3 \%$; $95 \% \mathrm{CI}$ $58.0-99.3 \%)$ and rehabilitation (89.5\%; 95\% CI 68.6-97.1\%) settings (27). However, gold standard assessments were conducted on only $68(21.9 \%)$ of the patients, and the lower limit of the $95 \%$ CI associated with sensitivity is lower (27) compared with the BBDST-R (71.9\% versus $84.9 \%)$. Suiter and Leder's study reported high sensitivity $(96.5 \%$; 95\% CI 94.9-97.6) and negative predictive value (97.9\%; 95\% CI 97.0-98.6) for aspiration (28). However, the study lacked blindness as the same investigator conducted both the screening and FEES (28). Sensitivity and negative predictive value of Clavé et al.'s instrument is $83.7-100 \%$ and $57.9-100 \%$, respectively (29). Wakasugi et al.'s instrument has $67-87 \%$ sensitivity and $84-95 \%$ negative predictive value (30). Neither of the two studies reported the associated CI. To summarize, all four studies contain certain shortcomings despite Kertscher et al.'s (3) conclusion regarding their methodological quality.

Implementation of the BBDST-R in practice. Screening is the essential first step in identifying risk of dysphagia that expedites referral to speech pathology for evaluation and treatment (31). As for the BBDST-R, it has already been implemented in clinical practice. For example, it is used in the neurology department of a large university hospital in Ostrava, Czech Republic (32). In the first year of its implementation (in 2013), a total of 1051 patients were screened (32). The result was negative in 662 of the patients (33). The remaining 389 patients (with positive screening result) were referred to a speech-language pathologist for detailed clinical examination of swallowing, who confirmed the diagnosis of dysphagia in 165 patients (33). Almost two thirds of these patients required rehabilitation of swallowing; only 12 were referred for FEES (33). In this particular hospital, implementation of the BBDST-R has promoted multidisciplinary collaboration and has facilitated the identification of patients with swallowing difficulties (32).

Limitations of the study. The first limitation is that the study was not entirely blinded as the physician performing FEES had access to bedside assessment results. In our opinion, this was important for ethical reasons as the physician needed the information to support high-quality care. The second limitation is that the period between bedside assessment and FEES was short ( $\leq 24$ hours) in "only" $96(90.6 \%)$ patients; in 10 other patients, it ranged from 2-22 days.

\section{Conclusion}

Our research has led to the development of the eightitem Brief Bedside Dysphagia Screening Test-Revised. In patients with neurological conditions, the BBDST-R has high sensitivity and negative predictive value. As part of the implications for practice, we recommend it for use in departments caring for patients with these conditions. It is very simple; therefore, it does not require extensive training of the personnel (e.g. nurses) involved in the screening. However, dysphagia screening is only the initial part of the diagnostic algorithm, and multidisciplinary collaboration remains of paramount importance. In other words, simple dysphagia screening does not replace the role of other health care providers whose expertise in the area of dysphagia assessment is much broader and deeper.

\section{Acknowledgements}

The study was partially financially supported by a grant from the Ministry of Health of the Czech Republic, IGA MZ NT 13725-4/2012.

\section{References}

1. Serra-Prat M, Hinojosa G, López D et al. Prevalence of oropharyngeal dysphagia and impaired safety and efficacy of swallow in independently living older persons. J Am Geriatr Soc 2011; 59: 186-187.

2. Park Y-H, Han H-R, Oh B-M et al. Prevalence and associated factors of dysphagia in nursing home residents. Geriatr Nurs 2013; 34: 212-217.

3. Kertscher B, Speyer R, Palmieri M. Bedside screening to detect oropharyngeal dysphagia in patients with neurological disorders: an updated systematic review. Dysphagia 2014; 29: 204-2012.

4. Speyer R, Kertscher B, Cordier R. Functional health status in oropharyngeal dysphagia. J Gastroenterol Hepatol Res 2014; 3: 1043-1048

5. Wilkins T, Gillies RA, Thomas AM, Wagner PJ. The prevalence of dysphagia in primary care patients: A HamesNet research network study. J Am Board Fam Med 2007; 20: 144-150.

6. Flowers HL, Silver FL, Fang J, Rochon E, Martino R. The incidence, co-occurrence, and predictors of dysphagia, dysarthria, and aphasia after first-ever acute ischemic stroke. J Commun Disord 2013; 46: 238-248.

7. Ho Y-H, Liu H-Y, Huang S-T. The prevalence and signs of dysphagia among stroke patients in rehabilitation units. J Nurs 2014; 61: 54-52.

8. Walker RW, Dunn JR, Gray WK. Self-reported dysphagia and its correlates within a prevalent population of people with Parkinson's disease. Dysphagia 2011; 26: 92-96.

9. Arribas L, Hurtós L, Milà R, Fort E, Peiró I. Predict factors associated with malnutrition from patient generated subjective global assessment (PG-SGA) in head and neck cancer patients. Nutr Hosp 2013; 28: 155-163.

10. Donovan NJ, Daniels SK, Edmiaston J, Weinhardt J, Summers D, Mitchell PH Dysphagia screening: State of the Art: Invitational Conference Proceedings From the State-of-the-Art Nursing Symposium, International Stroke Conference 2012. Stroke 2013; 44: e24-e31.

11. Bours GJJW, Speyer R, Lemmens J, Limburg M, de Wit R. Bedside screening test vs. videofluoroscopy or fibreoptic endoscopic evaluation of swallowing to detect dysphagia in patients with neurological disorders: systematic review. J Adv Nurs 2009; 65: 477-493.

12. Schepp SK, Tirschwell DL, Miller RM, Longstreth WT Jr. Swallowing screens after acute stroke: a systematic review. Stroke 2012; 43: 869-71.

13. Osawa A, Maeshima S, Tanahashi N. Water-swallowing test: screening for aspiration in stroke patients. Cerebrovasc Dis 2013; 35: 276-281.

14. Zuydam AC, Ghazali N, Lowe D, Skelly R, Rogers SN. Evaluations of the limitations of using the University of Washington Quality of Life swallowing domain alone to screen for patients in the routine clinical setting. Br J Oral Maxillofac Surg 2013; 51: e148-e154.

15. Cichero JAY, Heaton S, Bassett L. Triaging dysphagia: nurse screening for dysphagia in an acute hospital. J Clin Nurs 2009; 18: 1649-1659.

16. Steele CM, Molfenter SM, Bailey GL et al. Exploration of the utility of a brief 
swallow screening protocol with comparison to concurrent videofluoroscopy. Can J Speech Lang Pathol Audiol 2011; 35: 228-242.

17. Mandysova P, Škvrňáková J, Ehler E, Černý M. Development of the Brief Bedside Dysphagia Screening Test in the Czech Republic. Nurs Health Sci 2011; 13: 388-395.

18. Bochmann, F, Johnson Z, Azuara-Blanco A. Sample size in studies on diagnostic accuracy in ophthalmology: a literature survey. Br J Ophthalmol 2007; 91 898-900.

19. Carley S, Dosman S, Jones SR, Harrison M. Simple nomograms to calculate sample size in diagnostic studies. Emerg Med J 2005; 22: 180-181.

20. Flahault A, Cadilhac M, Thomas G. Sample size calculation should be performed for design accuracy in diagnostic test studies. J Clin Epidemiol 2005; 58:859-862.

21. Massey R, Jedlicka D. The Massey Bedside Swallowing Screen. J Neurosci Nurs 2002; 34: 252-260.

22. Trapl M, Enderle P, Nowotny M et al. Dysphagia bedside screening for acutestroke patients: the Gugging Swallowing Screen. Stroke 2007; 38: 2948-2952.

23. Rosenbek JC, Robbins JA, Roecker EB, Coyle JL, Wood JL. A penetration-aspiration scale. Dysphagia 1996; 11: 93-98.

24. Kraska-Miller M. Nonparametric statistics for social and behavioral sciences. Boca Raton, FL: CRC Press Taylor \& Francis Group, 2014: 260.
25. Schafer JL. Multiple imputation: A primer. Stat Methods Med Res 1999; 8: 3-15. 26. Lowry R. 2001-2013. Clinical calculator 1. (accessed December 31, 2013, at http://www.vassarstats.net)

27. Martino R, Silver F, Teasell R et al. The Toronto Bedside Swallowing Screening Test (TOR-BSST). Stroke 2009; 40: 555-561.

28. Suiter DM, Leder SB. Clinical utility of the 3-ounce Water Swallow Test. Dysphagia 2008; 23: 244-250.

29. Clavé P, Arreola V, Romea M, Medina L, Palomera E, Serra-Prat M. Accuracy of the volume-viscosity swallow test for clinical screening of oropharyngeal dysphagia and aspiration. Clin Nutr 2008; 27: 806-815.

30. Wakasugi $\mathrm{Y}$, Tohara $\mathrm{H}$, Hattori $\mathrm{F}$ et al. Screening test for silent aspiration at the bedside. Dysphagia 2008; 23: 364-370.

31. Daniels SK, Anderson JA, Willson PC. Valid items for screening dysphagia risk in patients with stroke: A systematic review. Stroke 2012; 43: 892-897.

32. Kaniová M, Kopecká B, Komínek P. Skríning poruch polykání a zkušenosti s jeho zaváděním ve FN Ostrava. Cesk Slov Neurol N 2014; 77/110 (Suppl.): S99. [in Czech]

33. Kaniová M, Kopecká B. Zkušenosti se screeningem poruch polykání na neurologické klinice FNO. Paper presented at certified course Poruchy polykání - diagnostika a léčba; Hradec Králové, Czech Republic; 2014 April 26. [in Czech]

Received: $24 / 03 / 2015$

Accepted in revised form: $11 / 05 / 2015$

\section{Corresponding author:}

Petra Mandysová, MSN, Ph.D., Faculty of Health Studies, University of Pardubice, Průmyslová 395, 53210 Pardubice, Czech Republic; e-mail: petra.mandysova@upce.cz 